\title{
Ameliorative Effect of Moringa Oleifera Biochemical Constituents on Blood Glucose Level of Streptozocin-Induced Diabetic Wistar Rats
}

\author{
Ibrahim Maina Hassan ${ }^{1}$, Bashir Saidu ${ }^{1}$, Ja'afaru Abdullahi Ishaq $^{1}$, Ashiru Dahiru', \\ Nafisat Abdulazeez ${ }^{1}$, Halima Ibrahim Yusuf ${ }^{1}$, Aliyu Habeeb Tosin ${ }^{1}$, Nicholas Nathaniel Pilau', \\ Adamu Abdul Abubakar', Muhammad Bashir Bello ${ }^{4}$ \\ ${ }^{1}$ Department of Veterinary Physiology and Biochemistry, Faculty of Veterinary Medicine, Usmanu Danfodiyo University, Sokoto, Nigeria \\ ${ }^{2}$ Department of Veterinary Medicine, Faculty of Veterinary Medicine, Usmanu Danfodiyo University, Sokoto, Nigeria \\ ${ }^{3}$ Department of Veterinary Surgery and Radiology, Faculty of Veterinary Medicine, Usmanu Danfodiyo University, Sokoto, Nigeria \\ ${ }^{4}$ Department of Veterinary Microbiology, Faculty of Veterinary Medicine, Usmanu Danfodiyo University, Sokoto, Nigeria
}

Email address:

ibrahim.hassan@udusok.edu.ng (I. M. Hassan)

\section{To cite this article:}

Ibrahim Maina Hassan, Bashir Saidu, Ja'afaru Abdullahi Ishaq, Ashiru Dahiru, Nafisat Abdulazeez, Halima Ibrahim Yusuf, Aliyu Habeeb Tosin, Nicholas Nathaniel Pilau, Adamu Abdul Abubakar. Muhammad Bashir Bello. Ameliorative Effect of Moringa Oleifera Biochemical Constituents on Blood Glucose Level of Streptozocin-Induced Diabetic Wistar Rats. Advances in Biochemistry. Vol. 9, No. 1, 2021 , pp. 6-10. doi: $10.11648 /$ j.ab.20210901.12

Received: March 19, 2020; Accepted: May 11, 2020; Published: February 23, 2021

\begin{abstract}
Medicinal plants are among the important source of potential therapeutic agents for diabetes. Therapeutic use of Moringa oleifera leaf extract has been practiced in management of diabetes because of its potential to decrease blood glucose and lipids concentration after ingestion. This study was conducted to evaluate the ameliorative effect of Moringa oleifera leaf extract on streptozocin induced diabetes Wistar rats. Initially, diabetes was induced by given streptozocin (STZ) intravenously followed by the treatments with different concentration of Moringa oleifera aqueous leaf extract. Fasting blood glucose level was measured using Accu-Chek glucometer after 24, 48, 72 and 97 hours post treatment. There is significance difference at 0.05 in blood glucose level between the control groups and the groups that were treated with the leaf extract after administration of STZ. Hence, this plant shows high potential to be used in the management of hyperglycaemia. The need for the toxicity study to evaluate it effect in body tissues and organs is recommended. There is need to study the phytochemical constituents to identify the bioactive lead compound with ameliorative effect.
\end{abstract}

Keywords: Ameliorative, Diabetes, Leaf Extract, Medicinal Plants, Moringa oleifera, Streptozocin, Wistar Rats

\section{Introduction}

Diabetes Mellitus is a group of metabolic diseases characterized by hyperglycemia resulting from defects in insulin secretion, insulin action, or both [1]. The chronic hyperglycaemia in diabetes mellitus disease results in severe damage, dysfunction, and failure of different tissues and organs especially eyes, kidneys, nerves, heart, and blood vessels [2]. Several patho-physiology changes occur in pathogenesis of diabetes mellitus. These include autoimmune destruction of the pancreatic $\beta$-cells with consequent insulin deficiency, abnormalities that result in resistance to insulin action, damaged to hepatic cells etc. [3]. The basis of the abnormalities in carbohydrate, fat, and protein metabolism in diabetes is due to insulin deficiency leading to inability of the sugar to be transported for it metabolism in the cells. This may be due to the complete absent or inadequate insulin secretion and/or diminished tissue responses to insulin at one or more points in the complex pathways of hormone action [4]. Impaired insulin secretion and or defects in insulin action have been observed in several patients with unknown causes. it is often unclear whether the aetiology of this diseases is specific, concurrently occur with other predisposing factor or inter currently as secondary to certain diseases [5]. In most cases, diabetes mellitus is characterized mainly with marked hyperglycemia. Other 
symptoms may include polyuria, polydipsia, weight loss, sometimes with polyphagia, and blurred vision [6]. Impairment of growth and susceptibility to certain infections accompanying chronic hyperglycemia has been reported. Acute, life-threatening consequences of uncontrolled diabetes due to hyperglycemia, keto acidosis or the non ketotic hyper osmolar syndrome have also been documented [7].

Moringa oleifera Lamarack belong to the genus Moringa, family Moringacea, the plant is use as important food commodity and enormous attention has been given to it due to its medicinal effects in tropics [8].

The leaf, fruit, flowers and immature pods of this tree are used as vegetable due to it highly nutritive value in many countries, particularly India, Pakistan, Philippines, Hawaii and many parts of Africa. Several health benefits of Moringa seeds, leaf or their extract as supplement has been reported [9]. The root, wood of this plant was shown to lower the deposition of stone forming constituents in the kidneys of calculogenic rats and reduced the elevated urinary oxalate as a result of ethylene glycol treatment [10]. Ameliorative effect of Moringa in induced liver fibrotic rats protect the liver from damage, symptoms of liver fibrosis was reverse few days after the used of this plant extract. The finding also reported alterations in serum aminotransferase activities and globulin level after CCl4- administration. Reductions of hepatic elevated hydroxyl proline content and myeloperoxidase activity were also observrd [11]. The leaf from this plant was used as good source of natural antioxidants and thus enhance the shelf-life of fat containing foods due to the presence of various antioxidant bioactive compounds such as ascorbic acid, flavonoids, phenolics and carotenoids and been reported to be a rich source of $\beta$-carotene, protein, vitamin $C$, calcium and potassium [12]. Diabetes mellitus is a metabolic condition that has been a leading cause of death in the whole world, especially among the less privileged population and rural areas due to lack of availability.

\section{Materials and Method}

\subsection{Materials}

Laboratory rats (Wistar rats), Moringaoleiferaleaf extract, Streptozocin (STZ), Glibenclamide, Accu-Chek Glucose meter (Glucometer), Glucometer or glucose strip, Gloves and Cotton wool, Razor blade, Oral cannula, Methylated spirit, Distilled water, Digital weighing balance, Syringes.

\subsubsection{Plant Collection and Identification}

Fresh leaf of Moringa oleifera found around Sokoto State Central Market was collected. The plant's leaf was identified and authenticated at the botany unit of Department of Biological Sciences in Usmanu Danfodiyo University, Sokoto, Nigeria.

\subsubsection{Plant Preparation}

The leaf was prepared according to the procedure described by Huang et al., 2017[13]. Briefly, the procedure involved drying the leaf in the laboratory to constant weight at room temperature. The leaf was then washed and grounded to semi powdered form using an electric blender. $5 \mathrm{~g}$ of the extract was mixed with $50 \mathrm{~mL}$ distilled water. The prepared solution was left to stand for two days and then filtered using muslin cloth.

\subsection{Animal}

Twenty four (24) Wistar rats weighing between (120-300g) of both sexes were used. They were obtained from the Animal House of the Department of Pharmacology and Clinical Pharmacy, Usmanu Danfodiyo University Teaching Hospital. They were kept in plastic cages under laboratory condition $\left(25-27^{\circ} \mathrm{C}\right)$ placed on standard feed and allowed free access to water.

Animal Grouping

Twenty four (24) Wistar rats were divided randomly into four groups (I-V) of six animals in each group $(n=6)$.

Group I: Control group (Normoglycemic animals)

Group II: Untreated diabetic group

Group III: Diabetic group that received Glibenclamide at 5 $\mathrm{mg} / 70 \mathrm{~kg} \mathrm{~b} / \mathrm{w}$

Group IV: Diabetic group that received Moringa leaves extract at $1000 \mathrm{mg} / \mathrm{kg} \mathrm{b} / \mathrm{w}$

\subsection{Induction of Experimental Diabetics}

Diabetes was induced by slow intravenous injection of Streptozocin solution (100 $\mathrm{mg} / \mathrm{kg}$ body weight) through the tail vein. The tail was cleaned with methylated spirit, cut at about $0.5 \mathrm{~cm}$. The diabetic state was confirmed 24 hours following administration of Streptozocinby by measuring the blood glucose level with Accu-Chek glucose meter (Glucometer). The blood from tail vessel was dropped on the sensor of the Accu-chek glucometer and the value for each rat was recorded.

\subsection{Statistical Analysis}

The data obtained were analyzed using microsoft office Excel 2007 and Statistical Package used was Graph prism version 5.0. Results were expressed as mean $\pm \mathrm{SD}$ and presented in tabular form. The result of serum biochemical analysis including creatinine, Urea, AST and ALT obtained from the experimental groups were compared with the of control groups using paired two-tailed student's t-test for matched samples and analysis of variance (ANOVA) was used for comparisons of three (3) or more values of the parameters in the various groups.

\section{Result}

\subsection{Result of Ameliorative Effect of Moringa oleifera Leaf Extract on Streptozocin-Induced Diabetes Wistar Rats After 24 Hours of Treatment}

Ameliorative effect of Moringa oleifera leaf extract on streptozocin-induced diabetes Wistar rats after 24 hours of treatment shows variation in blood glucose level. There is significance difference at $\mathrm{p}>0.01$ between the control groups (CTR) and the groups that received Streptozocin alone 
(DIABETIC ALONE). Significance difference at $\mathrm{p}>0.05$ was also observed between the control groups (CTR) and the groups that were induced with stz followed by the treatment with Moringa oleifera leaf extract (D+MLE) as well as the groups that were induced without treatment (DIABETIC ALONE) and the groups that were induced and treated with the standard drug glibenclamide $(\mathrm{D}+\mathrm{G})$ figure 1 .

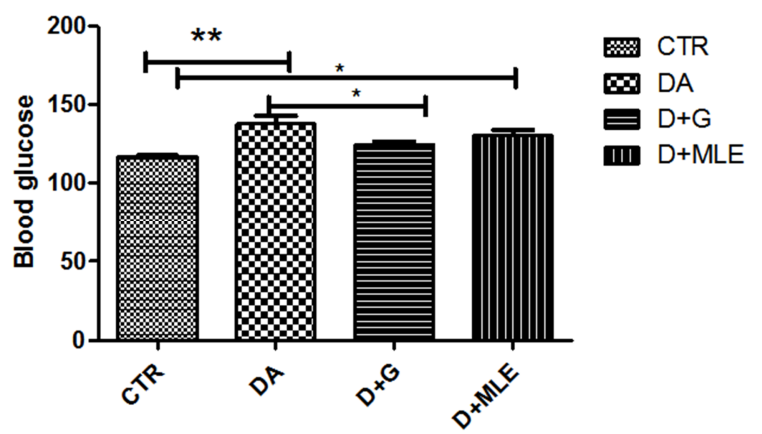

Figure 1. Ameliorative effect of Moringa oleifera (leaf) extract at 1000 $\mathrm{mg} / \mathrm{mL}$ on blood glucose level of experimentally diabetes induced Wister rats after 24 hours of treatment. There is variation in the blood glucose level between the experimentally exposed, exposed and treated with Moringa oleifera (leaf) extract at $1000 \mathrm{mg} / \mathrm{mL}$ as well as exposed and treated with glibenclamide group. Result is shown as concentration of blood glucose versus treatment and control. The values represent mean $\pm S D$ from two independent experiments with $(n=6)$.

\subsection{Result of Ameliorative Effect of Moringa oleifera Leaf Extract on Streptozocin-Induced Diabetes Wistar Rats After 48 Hours of Treatment}

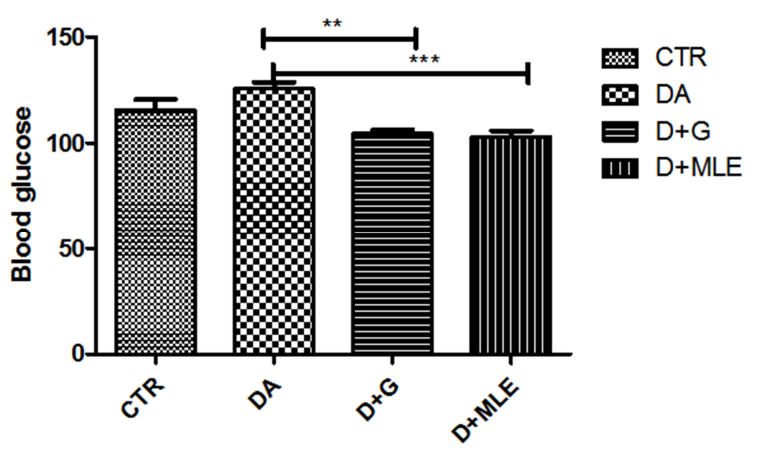

Figure 2. Ameliorative effect of Moringa oleifera (leaf) extract at 1000 $\mathrm{mg} / \mathrm{mL}$ on blood glucose level of experimentally diabetes induced Wister rats after 48 hours of treatment. There is variation in the blood glucose level between the experimentally exposed, exposed and treated with Moringa oleifera (leaf) extract at $1000 \mathrm{mg} / \mathrm{mL}$ as well as exposed and treated with glibenclamide group. Result is shown as concentration of blood glucose versus treatment and control. The values represent mean $\pm S D$ from two independent experiments with $(n=6)$.

Ameliorative effect of Moringa oleifera leaf extract on streptozocin-induced diabetes Wistar rats after 48 hours of treatment shows variation in blood glucose level. There is significance difference at $\mathrm{p}>0.01$ between the groups that received Streptozocin alone (DIABETIC ALONE) and the groups that were induced and treated with the standard drug glibenclamide $(D+G)$. Significance difference at $p>0.001$ was also observed between between the groups that received Streptozocin alone (DIABETIC ALONE) and the groups that were induced with stz followed by the treatment with Moringa oleifera leaf extract (D+MLE) figure 2.

\subsection{Result of Ameliorative Effect of Moringa oleifera Leaf Extract on Streptozocin-Induced Diabetes Wistar Rats After 72 Hours of Treatment}

Ameliorative effect of Moringa oleifera leaf extract on streptozocin-induced diabetes Wistar rats after 72 hours of treatment shows variation in blood glucose level. There is significance difference at $\mathrm{p}>0.001$ between the control groups (CTR) and the groups that were induced with stz followed by the treatment with Moringa oleifera leaf extract (D+MLE). Significance difference at $\mathrm{p}>0.001$ was also observed between control groups (CTR) and the groups and groups that were induced and treated with the standard drug glibenclamide $(\mathrm{D}+\mathrm{G})$. The result shows significance difference at $p>0.01$ between the groups that received Streptozocin alone (DIABETIC ALONE) and the groups that were induced with stz followed by the treatment with Moringa oleifera leaf extract (D+MLE) figure 3.

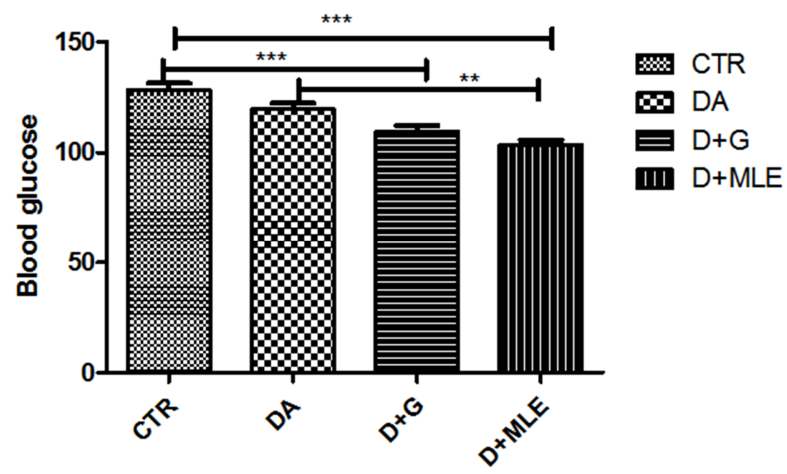

Figure 3. Ameliorative effect of Moringa oleifera (leaf) extract at 1000 $\mathrm{mg} / \mathrm{mL}$ on blood glucose level of experimentally diabetes induced Wister rats after 72 hours of treatment. There is variation in the blood glucose level between the experimentally exposed, exposed and treated with Moringa oleifera (leaf) extract at $1000 \mathrm{mg} / \mathrm{mL}$ as well as exposed and treated with glibenclamide group. Result is shown as concentration of blood glucose versus treatment and control. The values represent mean $\pm S D$ from two independent experiments with $(n=6)$.

\subsection{Result of Ameliorative Effect of Moringa oleifera Leaf Extract on Streptozocin-Induced Diabetes Wistar Rats After 96 Hours of Treatment}

Ameliorative effect of Moringa oleifera leaf extract on streptozocin-induced diabetes Wistar rats after 96 hours of treatment shows variation in blood glucose level. There is significance difference at $\mathrm{p}>0.001$ between the control groups (CTR) and the groups that were induced and treated with the standard drug glibenclamide $(D+G)$. The result shows significance difference at $p>0.01$ between the groups that received Streptozocin alone (DIABETIC ALONE) and the groups and groups that were induced and treated with the standard drug glibenclamide $(D+G)$. Significance difference at $p>0.001$ was also observed between the groups that were induced and treated with the standard drug glibenclamide $(D+G)$ the groups that were induced with stz followed by the 
treatment with Moringa oleifera leaf extract (D+MLE).

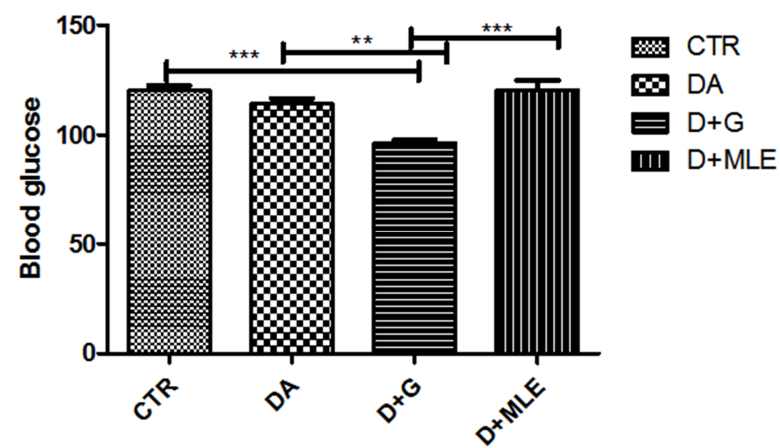

Figure 4. Ameliorative effect of Moringa oleifera (leaf) extract at 1000 $\mathrm{mg} / \mathrm{mL}$ on blood glucose level of experimentally diabetes induced Wister rats after 96 hours of treatment. There is variation in the blood glucose level between the experimentally exposed, exposed and treated with Moringa oleifera (leaf) extract at $1000 \mathrm{mg} / \mathrm{mL}$ as well as exposed and treated with glibenclamide group. Result is shown as concentration of blood glucose versus treatment and control. The values represent mean $\pm S D$ from two independent experiments with $(n=6)$.

\section{Discussion}

In this study, Moringa oleifera leaf extract was observed to lower glucose level after established diabetes induced by Streptozocin (STZ). Significance difference at $\mathrm{p}>0.01$ between the control groups and the groups that received STZ alone was observed. This is due to the increased blood sugar level in groups that received STZ only. STZ is an N-nitroso derivative of glucosamine extracted from Streptomyces acromogenes. STZ is a pancreatic-selective $\beta$-cell toxin that induces random, rapid and irreversible necrosis of $\beta$ cells. Necrosis of pancreatic cells results in increased blood sugar levels due to decrease or complete non secretion of insulin that transports sugar into cells and tissues for metabolism. This result agree with finding reported by Mostafavinia et al., 2016 [14] whose reported STZ to be considered as one of the most used diabetogenic compound for inducing diabetes in experimental animal models. Many reports indicated that STZ is capable of producing mild to severe types of diabetes that vary according to the selected dose, strain, and age of animals, nutritional status, and route of administration, along with other factors [15]. Moringa oleifera have been shown to have glucose lowering effect in hyperglycemic streptozocin induced diabetic rats. Although the mechanism of action have not been documented but possibly may be due to the stimulating effect of the crude extract on $\beta$-cells of pancreas [16]. This finding correspond with the finding reported by Onyagbodor \& Aprioku, which hows a significant decrease in the blood glucose levels between rat that received $250 \mathrm{mg} / \mathrm{kg}$ body weight of the extract of Moringa oleifera compared to the control that were place in normal commercial feed and tap water [17]. Decreased blood sugar levels of Moringa oleifera may be due to the present of several bioactive compound present in the leaf. A number of investigations have shown that flavonoids and a host of other secondary plant metabolites including arginine and glutamic acid present in Moringa oleifera leaf possess hypoglycemic effect in various animal models [18, 19]. Although the hypoglycemic effect of terpenoids appear to involve stimulation of pancreatic $\beta$-cells and subsequent secretion of preformed insulin [20]. Acute treatment of the 'control' Wistar rats with distilled water alone did not produced any change in the blood glucose concentration of either the fasted normal or the fasted STZtreated diabetic rats. However, aqueous plant extract, unlike insulin- an hypoglycemic agent, produced significant reduction in the blood glucose levels of fasted STZ treated diabetic rats. The plant extract examined in this study appears to act promptly and markedly only in fasted STZ treated diabetic rats. The hypoglycemic effect of Moringa oleifera appears to be most probably exerted via a mechanism that is different to that of insulin. A number of investigators have shown that coumarin, flavonoid, terpenoid and a host of other secondary plant metabolites including arginine and glutamic acids posses' hypoglycemic effects in various experimental animals model [21]. Hypoglycemic activity of Moringa oleifera leaf extract may probably be due to terpenoid present, which appears to be involved in the stimulation of the $\beta$-cells and the subsequent secretion of preformed insulin. One or more of the other chemical constituents of the plant especially flavonoid is also likely to have played a crucial role in the hypoglycemic action of the plant leaf extract.

\section{Conclusion}

The present study showed that aqueous leaf extract of Moringa oleifera possessed hypoglycemic properties in Streptozocin induced diabetic Wistar rats only, which suggest the presence of biologically active components which may be worth further investigation and elucidation. The effective hypoglycemic dose was found to be $1000 \mathrm{mg} / \mathrm{kg}$ body weight. Further studies are currently under way to isolate and characterized the active components of the crude extract of this plant.

\section{References}

[1] Ferdous, J., Ahmed, S., Laila, R., Islam, M. T., Rahaman, M. F., Snigdha, K. R.,... \& Sarkar, A. K., Determination of Insulin Secretory Defect and Insulin Sensitivity in Type 2 Diabetic Subjects in Bangladesh Mymensingh medical journal: MMJ 201625 (1): p. 109-118.

[2] Paari, E., \& Pari, L., Role Of Some Phytochemicals In The Management Of Diabetes Mellitus: A Review. Journal of Medical Practice and Review 20193 (04).

[3] Johar, D., Ahmed, S. M., Al-Dewik, N., Bahbah, E. I., Omar, N. H., Mustafa, M.,... \& Bernstein, L., Diabetes-Induced Proteome Changes Throughout Development. Endocrine, Metabolic \& Immune Disorders-Drug Targets (Formerly Current Drug Targets-Immune Endocrine \& Metabolic Disorders 2019. 19 (6): p. 732-743.

[4] Chattopadhyay, S., Samanta, S., Ganguly, S., Banerjee, J., \& Chanda, R., Types, Causes, Effect and Common Treatment of Diabetic Mellitus (DM), A Long Term Endocrine Metabolic Disorder:. A Review. Sustainable Humanosphere,, 202016 (1): p. 416-424. 
[5] McIntyre, H. D., Sacks, D. A., Barbour, L. A., Feig, D. S., Catalano, P. M., Damm, P., \& McElduff, A., Issues with the diagnosis and classification of hyperglycemia in early pregnancy.. Diabetes care 2016. 39 (1): p. 53-54.

[6] Pinheiro, M. M., Pinheiro, F. M. M., \& Trabachin, M. L., Dipeptidyl peptidase-4 inhibitors (DPP-4i) combined with vitamin D3: An exploration to treat new-onset type 1 diabetes mellitus and latent autoimmune diabetes in adults in the future International immunopharmacology 2018. 57: p. 11-17.

[7] Pasquel, F. J., Tsegka, K., Wang, H., Cardona, S., Galindo, R. J., Fayfman, M.,... \& Narayan, K. V., Clinical Outcomes in Patients With Isolated or Combined Diabetic Ketoacidosis and Hyperosmolar Hyperglycemic State: A Retrospective, Hospital-Based Cohort Study. 349-357. Diabetes Care 2020. 43 (2).

[8] Mahmood, K. T., Mugal, T., \& Haq, I. U., Moringa oleifera: a natural gift-A review. Journal of Pharmaceutical Sciences and Research, 2010. 2 (11): p. 775.

[9] Dogra, K. S., Chauhan, S., \& Jalal, J. S., Assessment of Indian medicinal plants for the treatment of asthma Journal of Medicinal Plants Research 2015. 9. (32): p. 851-862.

[10] El Rabey, H. A., Khan, J. A., Sakran, M. I., \& Al-Ghamdi, M. A., The Antioxidant Activity of Low Doses of Moringa Seeds (Moringa oleifera Lam.) in Hypercholesterolemic Male Rats Reactive Oxygen Species 20186 (17): p. 363-370.

[11] Farid, A. S., \& Hegazy, A. M., Ameliorative effects of Moringa oleifera leaf extract on levofloxacin-induced hepatic toxicity in rats Drug and chemical toxicolog 201948 (1): p. 345-55.

[12] Vergara-Jimenez, M., Almatrafi, M. M., \& Fernandez, M. L., Bioactive components in Moringa Oleifera leaves protect against chronic disease. Antioxidants 2017 6 (4): p. 91.

[13] Huang, Q., Zhao, W., Yan, A., \& Zhang, W., Applications of Reference Eextract in Determining the Characteristic Spectrum of Ginkgo Leaf Preparations.. Herald of Medicine,, 2017.. 36 (7): p. 794-797.
[14] Mostafavinia, A., Amini, A., Ghorishi, S. K., Pouriran, R., \& Bayat, M., The effects of dosage and the routes of administrations of streptozotocin and alloxan on induction rate of typel diabetes mellitus and mortality rate in rats. Laboratory animal research,, 2016. 32 (3): p. 160-165.

[15] Mustăciosu, C. C., Banciu, A., Rusu, C. M., Banciu, D. D., Savu, D., Radu, M., \& Radu, B. M., RNA-Binding Proteins $H u B, H u C$, and $H u D$ are Distinctly Regulated in Dorsal Root Ganglia Neurons from STZ-Sensitive Compared to STZResistant Diabetic Mice. International journal of molecular sciences 2019. 20 (8): p. 1965.

[16] Villarruel-López, A., López-de la Mora, D. A., VázquezPaulino, O. D., Puebla-Mora, A. G., Torres-Vitela, M. R., Guerrero-Quiroz, L. A., \& Nuño, K., Effect of Moringa oleifera consumption on diabetic rats.. BMC complementary and alternative medicine. 2018. 18 (1): p. 1-10.

[17] Onyagbodor, O. A., \& Aprioku, J. S., Moringa oleifera leaf extract inhibits diabetogenic effect of alloxan in rats.. IOSR Journal of Pharmacy, 2017. 7 (10): p. 07-12.

[18] Ghoneim, G., Ibrahim, F., \& El-gammal, R., HEPATOPROTECTIVE AND ANTIDIABITIC EFFECTS OF AQUEOUS Moringa oleifera LEAVES EXTRACT ON EXPERIMENTAL RATS.. Journal of Productivity and Development 2016. 21 (3): p. 203-219.

[19] Liyongo, C. I., Bongo, G. N., Ashande, C. M., Lufualabo, L. G., Gbolo, B. Z., Djoza, D. R.,... \& Mpiana, P. T., An Updated review on the Bioactivities and Phytochemistry of the Nutraceutical Plant Moringa oleifera Lam (Moringaceae) as valuable phytomedicine of multi-purpose.. Discovery Phytomedicine 2018. 5 (4): p. 52-63.

[20] Röder, P. V., Wu, B., Liu, Y., \& Han, W., Pancreatic regulation of glucose homeostasis.. Experimental \& molecular medicine 2016. 48 (3): p. e219-e219.

[21] Abd Eldaim, M. A., Shaban Abd Elrasoul, A., \& Abd Elaziz, S A., An aqueous extract from Moringa oleifera leaves ameliorates hepatotoxicity in alloxan-induced diabetic rats. Biochemistry and Cell Biology 2017. 95 (4): p. 524-530. 